\section{Revista 2020 \\ Med Vol. 28(1)}

Enero-Junio 2020
-

\title{
Epifisiólisis femoral proximal, una entidad desapercibida en atención primaria*
}

\author{
Christian José González Batecaa
}

\begin{abstract}
Resumen: La epifisiólisis femoral proximal o deslizamiento capital femoral es un trastorno de la cadera que se caracteriza por un desplazamiento de la epífisis sobre la metáfisis a través de la fisis (cartílago de crecimiento), quedando la epífisis posterior e inferior. Es una entidad relativamente frecuente en atención primaria y prehospitalaria que genera dolor y limitación de cadera; sin embargo, por su sintomatología vaga es usualmente infradiagnosticada o es diagnosticada tardíamente generando consecuencias a largo plazo. Si se tienen en cuenta los factores de riesgo asociados a una adecuada exploración física y el uso imágenes diagnósticas se lograría hacer un diagnóstico oportuno y una disminución de la tasa de complicaciones.
\end{abstract}

Palabras clave: epifisiólisis femoral; atención primaria; epífisis; metáfisis

Fecha de recepción: 15/04/2019 Fecha de aprobación: 10/03/2020.

Disponible en línea: 11/09/2020

Cómo citar: González Bateca CJ. Epifisiólisis femoral proximal, una entidad desapercibida en atención primaria. Rev. Med. [Internet]. 11 de septiembre de 2020 [citado 11 de septiembre de 2020];28(1). Disponible en: https://revistas.unimilitar.edu.co/index.php/rmed/article/view/4812

* Reporte de caso.

a MD. Universidad Autónoma de Bucaramanga, residente de especialización en Ortopedia y Traumatología, Fundación Universitaria Sanitas. Bogotá, Colombia Correo electrónico: christiangonzalezmd@hotmail.com ORCID: https://orcid.org/0000-0001-5540-5193 


\title{
Proximal Femoral Epiphysiolysis, an Unnoticed Entity in the Primary Care
}

\begin{abstract}
Proximal femoral epiphysiolysis or slipped capital femoral is a hip disorder characterized by a displacement of the epiphysis over the metaphysis through the physis (growth plate), leaving the epiphysis in a posterior and inferior position. It is a relatively frequent entity in primary and prehospital care that generates hip pain and limitation. However, due to its vague symptoms, it is usually underdiagnosed or diagnosed late, generating long-term consequences. If the risk factors associated to an adequate physical examination and the use of diagnostic images are taken into account, a timely diagnosis and a reduction in the rate of complications would be achieved.
\end{abstract}

Keywords: femoral epiphysiolysis; primary care; epiphysis; metaphysis

\section{Epifisiólise proximal do fêmur: uma entidade desapercebida em atenção primária}

Resumo: A epifisiólise proximal do fêmur ou escorregamento epifisário proximal do fêmur é um transtorno do quadril que é caracterizado por um deslocamento da epífise sobre a metáfise por meio da fise (placa de crescimento), ficando a epífise posterior e inferior. É uma entidade relativamente frequente em atenção primária e pré-hospitalar que gera dor e limitação do quadril; contudo, por sua sintomatologia vaga, é usualmente subdiagnosticada ou é diagnosticada tardiamente, o que leva a consequências em longo prazo. Se os fatores de risco associados com uma adequada exploração física e com o uso de imagens diagnósticas forem considerados, é possível realizar um diagnóstico oportuno e diminuir a taxa de complicações.

Palavras-chave: epifisiólise proximal do fêmur; atenção primária; epífise; metáfise 


\section{Clasificación}

Desde el punto de vista clínico la EFP puede clasificarse en función del tiempo de evolución, siendo crónica si persiste por más de tres semanas, aguda si dura menos de tres semanas y crónica reagudizada si los síntomas ocurren abruptamente con exacerbación del dolor e incapacidad para caminar, durante más de tres semanas. La variante crónica representa la mayoría de los casos en un $85 \%(4,19)$.

La clasificación descrita por Loder (20) es más útil y está determinada por los síntomas del paciente, específicamente por la tolerancia a la marcha, lo que establece de manera indirecta la estabilidad de la fisis; son estables el $90 \%$ (el paciente puede caminar) e inestables el resto (el paciente no puede caminar); dicha clasificación es práctica debido a que las estables son de mejor pronóstico comparadas con las inestables, que son de peor pronóstico y con mayor tasa de complicaciones $(1,2,15,19)$.

\section{Epidemiología}

La incidencia actual de EFP varía de 0,33 en 100.000 a 24,58 en 100.000 niños de ocho a quince años de edad, dependiendo del sexo y el origen étnico (19). Lehmann reportó una incidencia de EFP en Estados Unidos para niños de nueve y dieciséis años, de 10,80 casos / 100.000 niños (3), Perry reportó una incidencia de EFP en Reino Unido para niños de cero y dieciséis años de 4,8 casos / 100.000 niños (11). La incidencia fue 3,94 veces mayor en niños afroamericanos y 2,53 veces mayor en niños hispanos que en niños blancos $(3,4)$.

\section{Etiología y factores de riesgo}

La etiología de la EFP es controvertida; se piensa que es multifactorial, pero se han visto asociados factores mecánicos, obesidad, oleadas de crecimiento, trastornos endocrinos (hipotiroidismo, hipogonadismo, Síndrome Klinefelter). Todas ellas son alteraciones hormonales que disminuyen la resistencia de la fisis $(1,5,6,7,11,16)$.

\section{Presentación clínica}

Las manifestaciones clínicas dependerán de la estabilidad la EFP. Sin embargo, los síntomas más frecuentes son: cojera, dolor en la cadera, muslo o rodilla ipsilateral; alteraciones en el patrón de marcha, como marcha de pato o de Trendeleburg; además pueden cursar con un ángulo de progresión del pie en rotación externa. La cojera puede estar presente por varios meses y en estos casos el retraso del diagnóstico es frecuente; la demora promedio es de cinco meses entre el inicio de los síntomas y el diagnóstico (6), especialmente en aquellos pacientes que cursan con síntomas atípicos (11), como dolor en la rodilla o en el muslo distal. Esta presentación clínica de la EFP sigue siendo la dificultad más común en el diagnóstico de médicos locales (12). En el examen físico el miembro se encontrará en rotación externa durante flexión de la cadera (signo de Drehmann) (Figura 1) $(1,6,8,15,17,18)$.

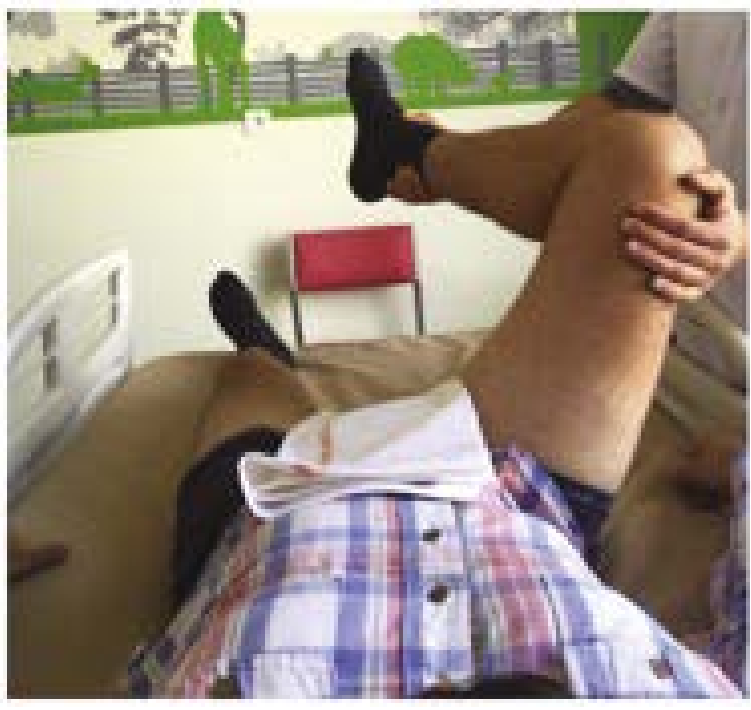

Figura 1. Signo de Drehmann, caracterizado por una rotación externa automática de la cadera mientras se flexiona.

Fuente: Klein, Haraux, Leroux y Gouron (6, p.3).

\section{Diagnósticos diferenciales}

Los diagnósticos diferenciales de un paciente joven que presenta dolor de cadera $(1,18)$. Existen múltiples diagnósticos diferenciales de dolor de cadera y cojera en población pediátrica, sin embargo, cada uno con características particulares de acuerdo a la edad, hallazgos clínicos y radiológicos que permiten hacer una aproximación diagnostica adecuada (Tabla 1). 


\begin{tabular}{|c|c|c|c|c|}
\hline Enfermedad & Edad & Hallazgos clínicos & Frecuencia & Diagnóstico \\
\hline $\begin{array}{l}\text { Avulsión traumática } \\
\text { apofisaria }\end{array}$ & 12 a 25 & $\begin{array}{l}\text { Dolor después de un movimiento } \\
\text { fuerte repentino }\end{array}$ & A menudo & Historia de trauma radiografía \\
\hline Apofisitis de la cadera & 12 a 25 & $\begin{array}{l}25 / 5.000 \\
\text { Dolor de cadera relacionado con la } \\
\text { actividad }\end{array}$ & A menudo & $\begin{array}{l}\text { Historia de uso excesivo; } \\
\text { radiografía }\end{array}$ \\
\hline Sinovitis transitoria & $<10$ & Cojera o dolor de cadera & A menudo & $\begin{array}{l}\text { Radiografía; laboratorios; } \\
\text { ecografía }\end{array}$ \\
\hline Fractura & $\begin{array}{l}\text { Todas las } \\
\text { edades }\end{array}$ & Dolor después de un trauma & Ocasionalmente & Historia de trauma radiografía \\
\hline EFP & 10 a 15 & $\begin{array}{l}\text { Dolor en cadera, ingle, muslo o } \\
\text { rodilla; cojeando }\end{array}$ & Ocasionalmente & $\begin{array}{l}\text { Radiografía de cadera bilateral } \\
\text { (ap y lateral) }\end{array}$ \\
\hline $\begin{array}{l}\text { Enfermedad de } \\
\text { Perthes }\end{array}$ & 4 a 9 & $\begin{array}{l}\text { Dolor vago en la cadera, disminución } \\
\text { de la rotación interna de la cadera }\end{array}$ & Infrecuente & Radiografía de cadera o rmn \\
\hline Artritis Séptica & $\begin{array}{l}\text { Todas las } \\
\text { edades }\end{array}$ & Fiebre, cojera, dolor de cadera & Infrecuente & $\begin{array}{l}\text { Radiografía; laboratorios, } \\
\text { artrocentesis }\end{array}$ \\
\hline
\end{tabular}

Tabla 1. Diagnósticos diferenciales en dolor de cadera

Fuente: (1).

\section{Hallazgos radiológicos}

La radiografía comparativa anteroposterior (AP) y lateral de las caderas es fundamental en el abordaje de un paciente con cojera; específicamente en EFP existen hallazgos radiográficos que nos permiten orientar hacia un diagnóstico; en la proyección AP se puede visualizar una doble densidad radiográfica creada por la epífisis que se desplaza posteriormente y se superpone a la parte medial de la metáfisis conocida como el signo de Steel (Figura 2 B); También se puede apreciar un ensanchamiento relativo de la fisis (Figura $2 \mathrm{C}$ ) o una disminución de la altura de la epífisis (Figura 2 D). Finalmente, al trazar una línea en el borde superior del cuello femoral (línea de Klein), en una cadera normal esta debería cruzar la porción superior de la epífisis ( $\mathrm{Fi}$ gura $2 \mathrm{~A}$ ); en la EFP, esta línea no pasa por la epífisis o la cruza en menor grado que en la cadera sana (Signo de Trethowan) (Figura 2 E) $(1,9,15,17,18)$.
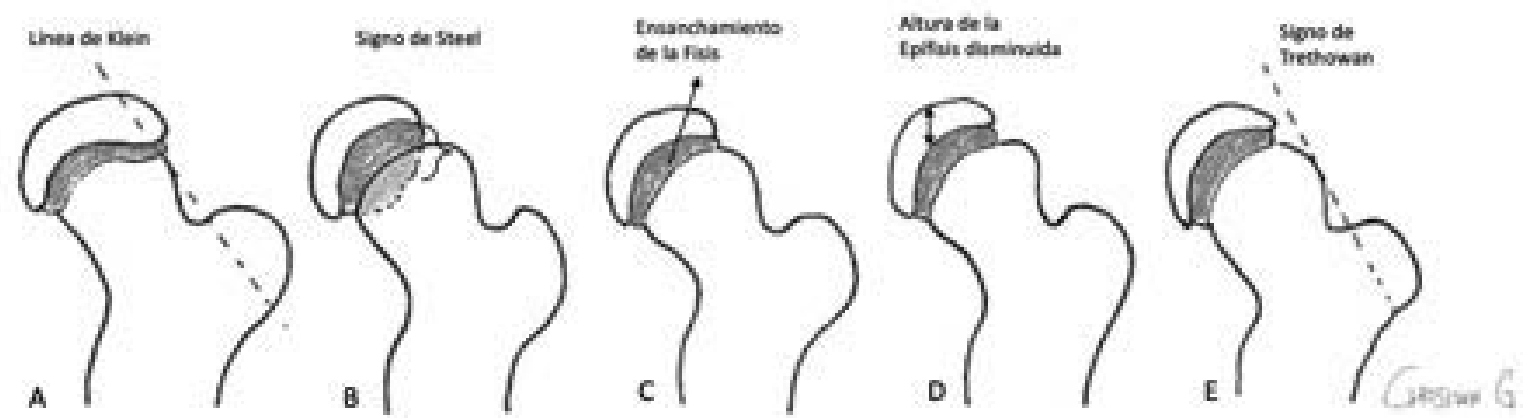

Figura 2. Hallazgos Radiológicos en EFP.

Fuente: elaboración propia con base en (1). 
La EFP también puede clasificarse radiológicamente según el porcentaje de desplazamiento de la cabeza femoral respecto al cuello, siendo leve cuando el desplazamiento es menor al $33 \%$, moderado cuando el desplazamiento está entre el $33 \%$ y el $50 \%$ y severo si tenemos un desplazamiento mayor al $50 \%$ (Figura 3). El otro método de clasificación es el descrito por Southwick, el cual depende de la magnitud del ángulo epifisario-metafisario en la proyección lateral; este se obtiene trazando una línea paralela al cartílago de crecimiento de la cabeza femoral. Se dibuja una perpendicular a esta línea y luego se traza una línea que sigue el eje del cuello femoral. El ángulo formado por estas dos últimas líneas determina el grado de desplazamiento comparándose con la cadera sana, y puede clasificarse como leve cuando el ángulo es menor a $30^{\circ}$; moderado, si el ángulo está entre $30^{\circ}$ y $50^{\circ}$; y grave, cuando es mayor a $50^{\circ}$ (Figura 4$)(15,17)$.

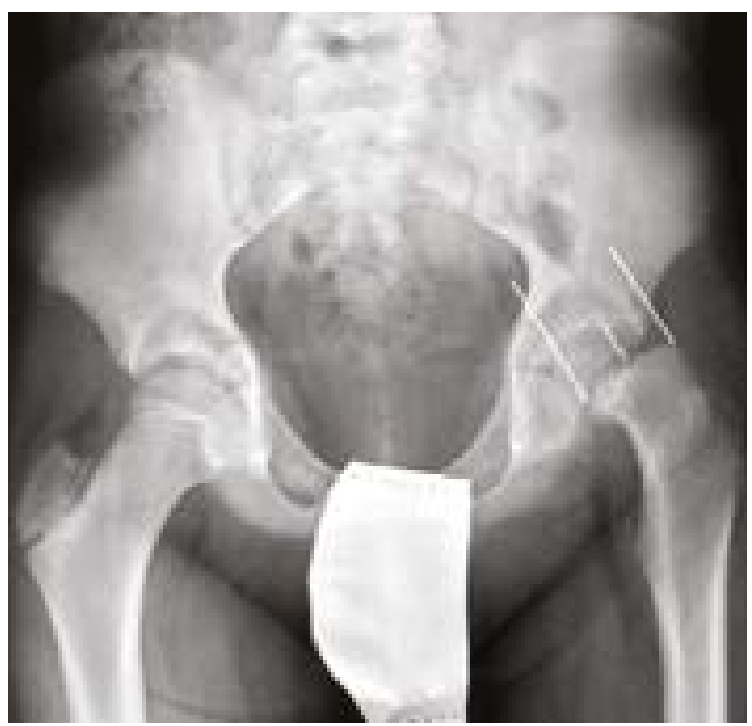

Figura 3. Radiografía A-P de pelvis con el porcentaje de desplazamiento de la epífisis sobre la anchura de la metáfisis.

Fuente: Martínez-Álvarez, Martínez-González, Miranda Gorozarri, Abril y Epeldegui (15, p.4).

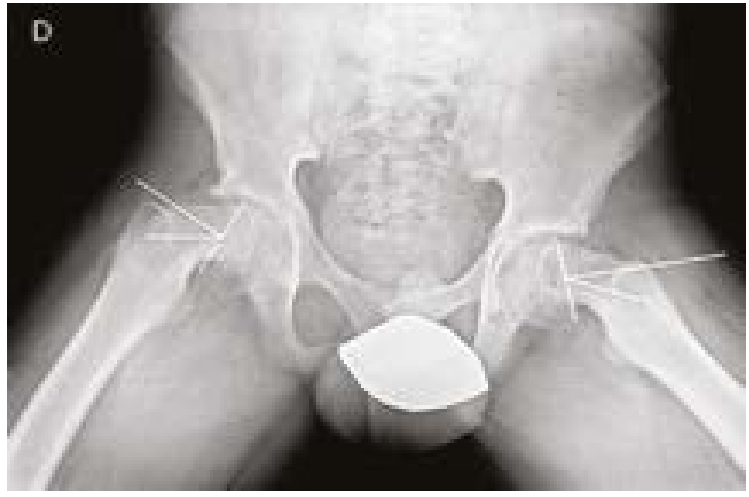

Figura 4. Radiografía axial de ambas caderas. Epifisiólisis de $36 \circ$ en cadera derecha y $27 \circ$ en cadera izquierda.

Fuente: Martínez-Álvarez et al. (15, p.4).

\section{Tratamiento}

La EFP es una urgencia quirúrgica y sus objetivos principales son detener y evitar la progresión del deslizamiento de la epífisis y prevenir complicaciones. El tratamiento es quirúrgico y dependerá del grado de deslizamiento epifisario y el tiempo de evolución del cuadro clínico.

El tratamiento estándar para EFP estable es la fijación in situ con tornillos. La reducción cerrada no está indicada para casos, debido a que la continuidad entre la epífisis y la metáfisis no se ha interrumpido, el intento de reducción puede fallar y existe el riesgo de lesiones iatrogénicas. En EFP estable se pueden emplear también osteotomías para mejorar la posición de la epífisis en relación con el acetábulo. Estos métodos tienen una alta tasa de éxito a largo plazo, con un riesgo mínimo de complicaciones $(18,21)$.

El caso de EFP inestable es mucho más severa ya que la epífisis se separa de la metáfisis comprometiendo la irrigación de la epífisis con el riesgo de necrosis avascular ulterior. Las alternativas de tratamiento y el tiempo de cirugía son temas de controversia; sin embargo, el procedimiento Dunn modificado es una opción terapéutica que ha ganado popularidad y consiste en una luxación quirúrgica de la cadera que ayuda a restaurar la alineación del fémur proximal; esta ha demostrado resultados favorables con respecto a las tasas 
de necrosis avascular, resultado funcional y el desarrollo de la osteoartritis radiográfica $(21,22)$.

El tratamiento preventivo de la cadera contralateral no se recomienda sistemáticamente; es útil solo para pacientes con endocrinopatía, déficit metabólico o aquellos en los que el monitoreo es difícil $(6,10)$.

\section{Complicaciones}

Complicaciones habituales: la condrólisis y la necrosis avascular de la cabeza femoral (13). La necrosis avascular puede presentarse hasta en un $50 \%$ de los pacientes con EFP inestable; se observa en las lesiones agudas no reducidas, en pacientes sometidos a múltiples intentos de reducción o luego de hacer osteotomías como medida de tratamiento. La necrosis conduce a la deformación de la cabeza femoral generando dolor en la cadera, rigidez articular, osteoartritis, resultando a largo plazo en artroplastia total de cadera $(6,18)$.

La condrólisis consiste en una necrosis aguda del cartílago de la epífisis femoral que se manifiesta con dolor y rigidez articular progresiva; tiene una incidencia aproximada del 1,8 \% al $55 \%$; puede ser secundaria a una penetración intraarticular del material de osteosíntesis en la cabeza femoral, o en algún factor autoinmune asociado $(1,6,15)$.

En ambas condiciones el tratamiento consiste en preservar la movilidad y restringir el apoyo completo con muletas, fisioterapia, analgesia. No obstante, los pacientes con condrólisis tienen mejor pronóstico a largo plazo (15).

El pinzamiento fémoro-acetabular también se encuentra descrito como una complicación y hace referencia a un cambio en la morfología; en el cuello y la cabeza femorales que lesionan el cartílago articular y el labrum progresivamente, causando dolor, limitación y degeneración osteoartrósica a mediano y largo plazo (4). Esto representa que la EFP sea una de las causas más comunes de reemplazo de cadera tanto en la adolescencia como en el adulto joven (14). El diagnóstico y tratamiento se salen de los propósitos del presente artículo $(15,18)$.

\section{Conclusión}

La EFP es una causa importante de discapacidad en niños y adultos jóvenes que representa no solo un desafío importante para el ortopedista, sino también para el sistema de atención primaria de salud. Partiendo de este punto el médico de atención primaria debe tener un alto índice de sospecha considerando variables como la edad, sexo, raza, comorbilidades como la obesidad y endocrinopatías. Estos factores, asociados a una adecuada anamnesis, exploración física y finalmente con el apoyo de imágenes diagnósticas, le permitirán un diagnóstico oportuno, derivación y posterior intervención temprana que disminuirá la tasa de complicaciones generadas por un diagnóstico erróneo o tardío.

\section{Referencias}

[1] Peck D. Slipped capital femoral epiphysis: diagnosis and management [Internet]. Am Fam Physician. 2010;82(3):258-262. Disponible en: https://pubmed. ncbi.nlm.nih.gov/20672790/

[2] Loder RT, Starnes T, Dikos G, Aronsson D. Demographic predictors of severity of stable slipped capital femoral epiphyses [Internet]. JBJS January. 2006;88(1):97-105. DOI: https://doi.org/ 10.2106/00004623-200601000-00012

[3] Lehmann CL, Arons RR, Loder RT, Vitale MG. The epidemiology of slipped capital femoral epiphysis: an update [Intyernet]. J Pediatr Orthop. 2006;26(3):286-290. DoI: https://doi.org/10.1097/01. bpo.0000217718.10728.70

[4] Iwinski HJ Jr. Slipped capital femoral epiphysis [Internet]. Current Opinion in Orthopaedics: December. 2006;17(6):511-516. DOI: https://doi.org/10.1097/01. bco.0000247364.51879.16

[5] Barrios C, Blasco MA, Blasco MC, Gascó J. Posterior sloping angle of the capital femoral physis: a predictor of bilaterality in slipped capital femoral epiphysis [Internet]. J Pediatr Orthop. 2005;25(4):445-449. DOI: https://doi.org/10.1097/01.bpo.0000158811.29602.a5

[6] Klein C, Haraux J, Leroux R. Gouron. Épiphysiolyse fémorale supérieure [Internet]. Archives de pédiatrie. 24;(3):301-305. DoI: https://doi.org/10.1016/j.arcped.2016.12.011 
[7] Novais EN, Millis MB. Slipped Capital Femoral Epiphysis: Prevalence, Pathogenesis, and Natural History [Internet]. Clin Orthop Relat Res. 2012; 470:34323438. DoI: https://doi.org/10.1007/s11999-012-2452-y

[8] Jochen G, Gerstner JB. Semiología del Aparato Locomotor. Bogotá: Editorial Medica Celsus; 2011. 303 p.

[9] Gekeler J. Radiology of Adolescent Slipped Capital Femoral Epiphysis [Internet]. Oper Orthop Traumatol. 2007;19(4):329-344. DoI: https://doi.org/10.1007/ s00064-007-1214-6

[10]Riad J, Bajelidze G, Gabos PG. Bilateral slipped capital femoral epiphysis: predictive factors for contralateral slip [Internet]. J Pediatr Orthop. 2007;27(4):411-414. DoI: https://doi.org/10.1097/01. bpb.0000271325.33739.86

[11] Perry DC, Metcalfe D, Costa ML, et al. A nationwide cohort study of slipped capital femoral epiphysis [Internet]. Archives of Disease in Childhood. 2017;102:1132-1136. DoI: https://doi.org/10.1136/archdischild-2016-312328

[12]Rahme D, Comley A, Foster B, Cundy P. Consequences of diagnostic delays in slipped capital femoral epiphysis [Internet]. J Pediatr Orthop B. 2006;15(2):93-97. DoI: https://doi.org/10.1097/01. bpb.0000188251.24771.c9

[13] Lubicky JP. Chondrolysis and avascular necrosis: complications of slipped capital femoral epiphysis [Internet]. J Pediatr Orthop B. 1996;5(3):162-167. Dor: https:// doi.org/10.1097/01202412-199605030-00005

[14] National joint registry of England and wales. 9th Annual Report [Internet]. 2012. Disponible en: https:// www.hqip.org.uk/wp-content/uploads/2018/02/national-joint-registry-9th-annual-report-2012.pdf

[15] Martínez-Álvarez S, Martínez-González C, Miranda Gorozarri, C, Abril JC, Epeldegui T. Epifisiolisis de la cabeza femoral [Internet]. Revista Española de Cirugía Ortopédica y Traumatología. 2012;56(6):506-514. DOI: https://doi.org/10.1016/j.recot.2012.07.004

[16] Rosselli C. Ortopedia Infantil. En Colombia 2012. Bogotá: Editorial Médica Panamericana.419-425.

[17] Georgiadis AG, Zaltz I. Slipped Capital Femoral Epiphysis [Internet]. Pediatric Clinics of North America. 2014;61(6):1119-1135. DoI: https://doi.org/10.1016/j. pcl.2014.08.001

[18] Peck DM, Voss LM, Voss TT. Slipped Capital Femoral Epiphysis: Diagnosis and Management [Internet]. Am Fam Physician. 2017;95(12):779-784.
[19]Aprato A, Conti A, Bertolo F, Massè A. Slipped capital femoral epiphysis: current management strategies [Internet]. Orthop Res Rev. 2019;11:47-54. DoI: https://doi.org/10.2147/ORR.S166735

[20]Loder RT, Richards BS, Shapiro PS, et al. Acute slipped capital femoral epiph- ysis: the importance of physeal stability [Internet]. J Bone Joint Surg Am. 1993;75(8): 1134-1140. DoI: https://doi.org/10.2106/00004623199308000-00002

[21]Otani T, Kawaguchi Y, Marumo K. Diagnosis and treatment of slipped capital femoral epiphysis: Recent trends to note [Internet]. J Orthop Sci. 2018;23(2):220-228. DOI: https://doi.org/10.1016/j. jos.2017.12.009

[22]Tannast M, Jost LM, Lerch TD, Schmaranzer F, Ziebarth K, Siebenrock KA. The modified Dunn procedure for slipped capital femoral epiphysis: the Bernese experience [Internet]. J Child Orthop. 2017;11(2):138-146. DoI: https://doi.org/10.1302/1863-2548-11-170046 